\title{
Length of Maximal Common Subsequences
}

\author{
Kim S. Larsen \\ kslarsen@daimi.aau.dk \\ Computer Science Department, Aarhus University \\ Ny Munkegade, 8000 Aarhus C, Denmark
}

October 1992

\begin{abstract}
The problem of computing the length of the maximal common subsequences of two strings is quite well examined in the sequential case. There are many variations, but the standard approach is to compute the length in quadratic time using dynamic programming. A lineartime parallel algorithm can be obtained via a simple modification of this strategy by letting a linear number of processors sweep through the table created for the dynamic programming approach.

However, the contribution of this paper is to show that the problem is in NC. More specifically, we show that the length of the maximal common subsequences of two strings $s$ and $t$ can be computed in time $O(\log |s| \cdot \log |t|)$ in the CREW PRAM model and in time $\Theta(\min (\log |s|$, $\log |t|))$ in the COMMON CRCW PRAM model.
\end{abstract}

\section{Introduction}

A subsequence of a string $s$ is any string, which can be created from $s$ by deleting some of the elements. More precisely, if $s$ is the string $s_{1} s_{2} \cdots s_{k}$ then $s_{i_{1}} s_{i_{2}} \cdots s_{i_{p}}$ is a subsequence of $s$ if $\forall j \in\{1, \ldots, p\}: i_{j} \in\{1, \ldots, k\}$ 
and $\forall j \in\{1, \ldots, p-1\}: i_{j}<i_{j+1}$. For example, parle is a subsequence of parallel.

Consider two fixed strings $s=s_{1} s_{2} \cdots s_{k}$ and $t=t_{1} t_{2} \cdots t_{m}$. Among the strings which are subsequences of both $s$ and $t$, there will be some of maximal length. Such subsequences are called maximal common subsequences.

For example, parallel and peal have maximal common subsequences pal and pel. However, the length of the maximal common subsequences is unique; in this case, it is three. Let count $(s, t)$ denote the length of the maximal common subsequences of $s$ and $t$.

The problem of computing count $(s, t)$ is quite well examined in the sequential case. The solutions in the literature are variations over the basic dynamic programming approach of filling out a table with count values for prefixes of the argument strings. In the next section, we present one of these algorithms, which has time complexity $\Theta(|s| \cdot|t|)$. Faster algorithms can be obtained for some special cases such as: short second argument, nearly identical strings, etc. A summary of these results can be found in $[1,4]$. In this paper, the focus is on the parallel time complexity.

All algorithms presented here will be given using $\mathrm{C}$. The code will be almost complete. We will leave out some of the most trivial details, though. When describing parallel algorithms, a few extra primitives are needed. We assume that processes can be declared using the keyword process, and that each processor has an id variable. Processors are assumed to be numbered from one. For synchronization purposes, we use the keyword await followed by a boolean expression. This could have been written using a busy loop. However, we prefer to high-light the synchronization points by using a special keyword. Finally, variables not declared in the processes are assumed to be global.

\section{A Sequential Solution}

For completeness, we now describe one of the standard sequential solutions for computing count $(s, t)$. We assume that $s$ is in $s[1], s[2], \ldots, s[k]$ and that $t$ is in $t[1], t[2], \ldots, t[m]$. A table is created and the value count $\left(s_{1} s_{2} \ldots s_{i}\right.$, $\left.t_{1} t_{2} \ldots t_{j}\right)$ will be stored in table $[i][j]$. 
It turns out to be convenient to represent explicitly the fact that the value of count $(s, t)$ is zero if one of the strings is empty. The zero-entries will be used for that. This decision will cut down on special cases in the rest of the algorithm. This border is first filled out, and then the remaining entries are computed from top to bottom and from left to right.

This formulation of the algorithm is from [2], except that we are not concerned with the space complexity issues. The algorithm has time complexity $\Theta(|s| \cdot|t|)$.

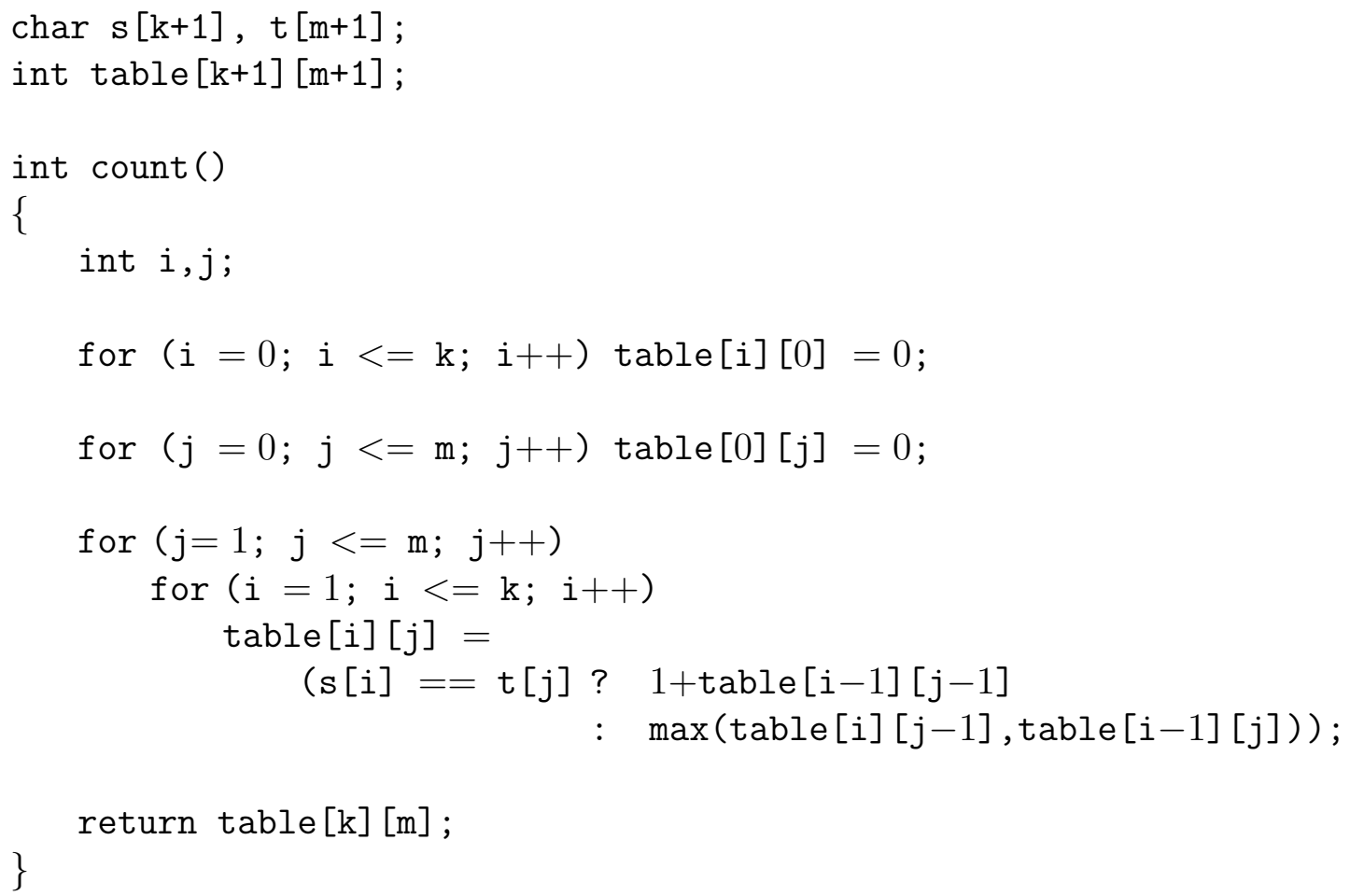

\section{Parallel Solutions}

The dynamic programming solution presented in the previous section is the starting point for the parallel versions. In this section, we first present the most obvious generalization. This solution is included primarily in order to present the framework on a simple example. The second solution is more 
involved. Here, we are trying to get the asymptotic parallel time complexity down as far as possible.

\subsection{A Simple Parallel Solution}

An easy observation from the sequential case is that the value of table $[i][j]$, independently of whether or not $s[i]$ equals $t[j]$, is computed from table entries table $\left[i^{\prime}\right]\left[j^{\prime}\right]$, where $i^{\prime}+j^{\prime}<i+j$. This means that a parallel version can be designed by letting a number of processors sweep diagonally down the table.

As in the sequential dynamic programming solution, we use one row and one column to explicitly represent the fact that a count involving an empty string gives zero. The processors share the responsibility for initializing these extra entries.

The "sweep" now works as follows. We use $m$ processors numbered from one. Processor $j$ will be responsible for computing all the entries in the $j$ th row. In the first step, processor 1 computes table[1][1]. In the second step, processor 1 computes table[2][1] while processor 2 computes table[1][2]. In the third step, the processors 1, 2, and 3 compute the entries table[3][1], table[2][2], and table[1][3], respectively. Proceeding like this, the whole table will be filled out in $|s|+|t|-1$ steps. Notice that just as some processors start late, some processors finish early.

In each step, each processor performs a constant amount of work. This can easily be bounded. We assume that the global variable step is incremented by a designated processor using this bound. All $m$ processors have the same code, which depend on their $i d$ variable:

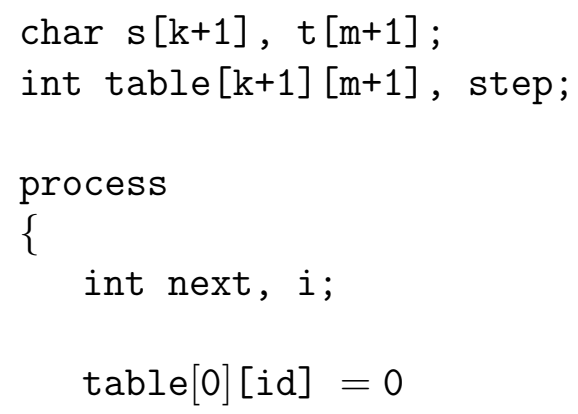




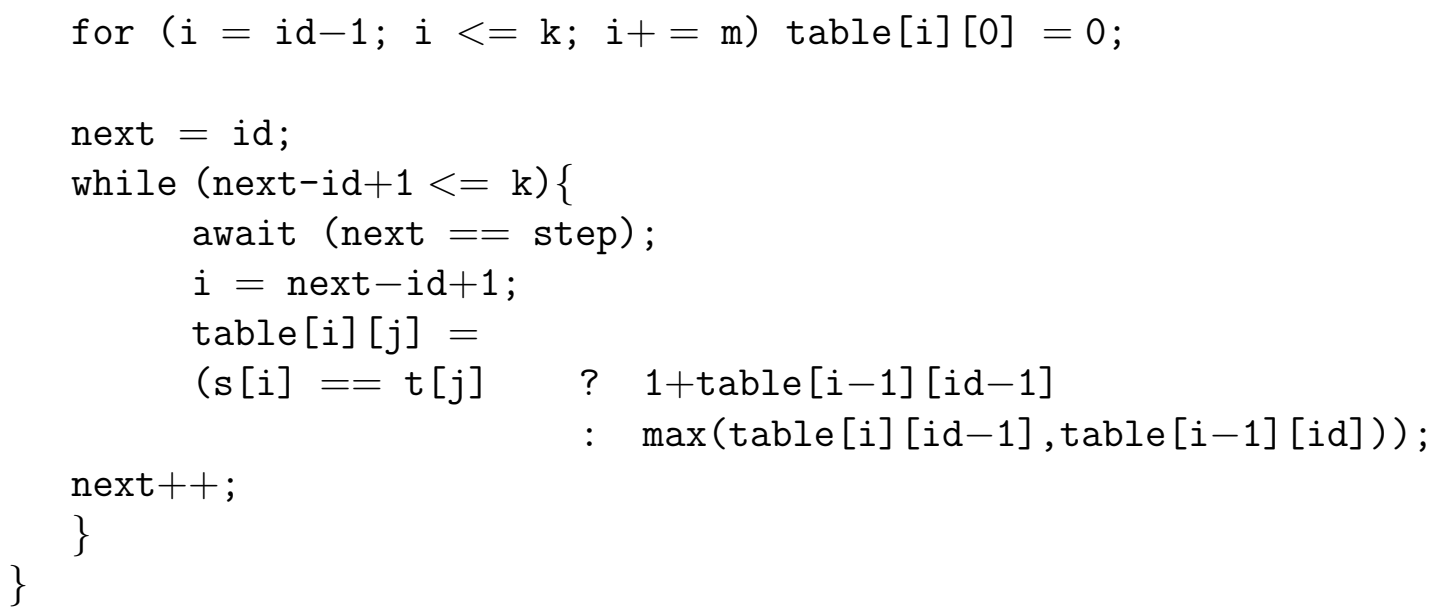

The result of the computation can be found in table $[k][m]$. We have demonstrated that count $(s, t)$ can be computed in time $\Theta(|s|+|t|)$ on a CREW PRAM (or even an EREW PRAM).

\subsection{A Fast Parallel Solution}

By only storing count values, as we did in the previous section, it does not seem possible to get below $\Theta(|s|+|t|)$ because of the dependencies in the table. In the following, we store more information. Each table entry, table $[i][j]$, will now be a table of size $|t|+1$ instead of simply an integer.

The processors contain a loop, which will be executed at most log $|s|+1$ times. We assume that $|s|$ is a power of two (if not, then we can always extend $s$ using a special symbol not appearing elsewhere in the strings; this cannot affect the value of count). As in the previous algorithm, a global variable step is used to count the number of iterations of these loops. At a certain point, given the value of step, we are only interested in the following substrings of $s$ :

$$
s_{1} \cdots s_{2^{s t e p}}, s_{2^{s t e p}+1} \cdots s_{2 \cdot 2^{\text {step }}}, s_{2 \cdot 2^{\text {step }}+1} \cdots s_{3 \cdot 2^{\text {step }}} \cdots,|s| \text {. }
$$

In that round, only the entries table $[i][j][v]$, where $i \in\left\{2^{\text {step }}, 2 \cdot 2^{\text {step }}, 3\right.$. $\left.2^{\text {step }}, \ldots,|s|\right\}$ are maintained. 
The basic idea is that $p=$ table $[i][i][v]$ should be the maximal index (giving rise to the shortest substring of $t$ ) such that

$$
\operatorname{count}\left(s_{i-2^{\text {step }}+1} \cdots s_{i}, t_{p+1} \cdots t_{j}\right)=v
$$

Notice that the substring of $t$ starts with $t_{p+1}$ rather than with $t_{p}$. This results in a more elegant algorithm, as we need not worry about $t_{p}$ being matched with two elements from $s$, when combining the values for two adjacent substrings of $s$ into a value for the concatenation.

In the algorithm, we assign a processor to each table $[i][j][v]$ entry. Each of these processors controls their own collection of processors to compute the maxima they need. Exactly how many processors are needed for this depends on how fast we want the maxima computed and in which model. We return to the complexity issues later.

We assume that in each step, all processors have time to read all their entries before any processor writes. Apart from computing maxima, the computation strategy of which we have not specified, we are within the CREW model of computation, as only processor $(i, j, v)$ writes in table $[i][j][v]$.

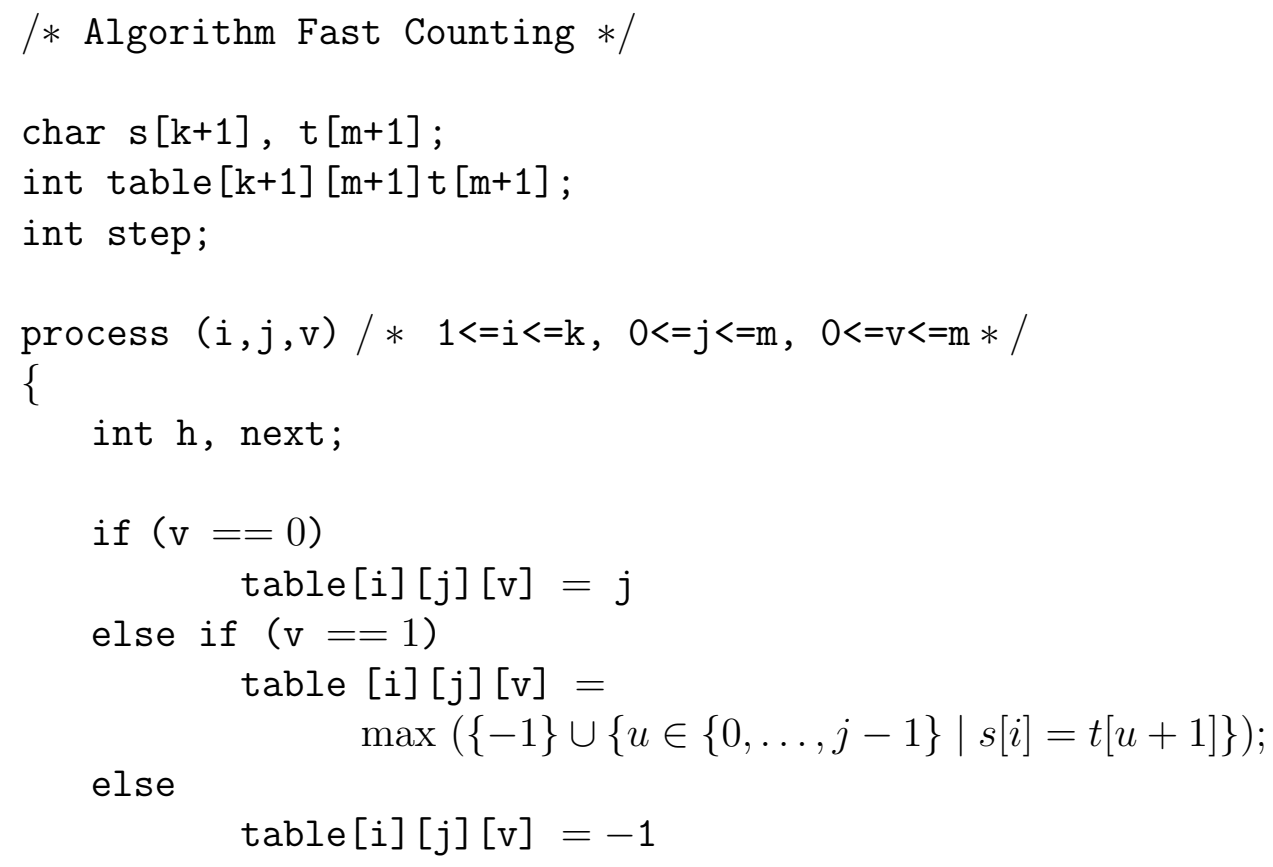




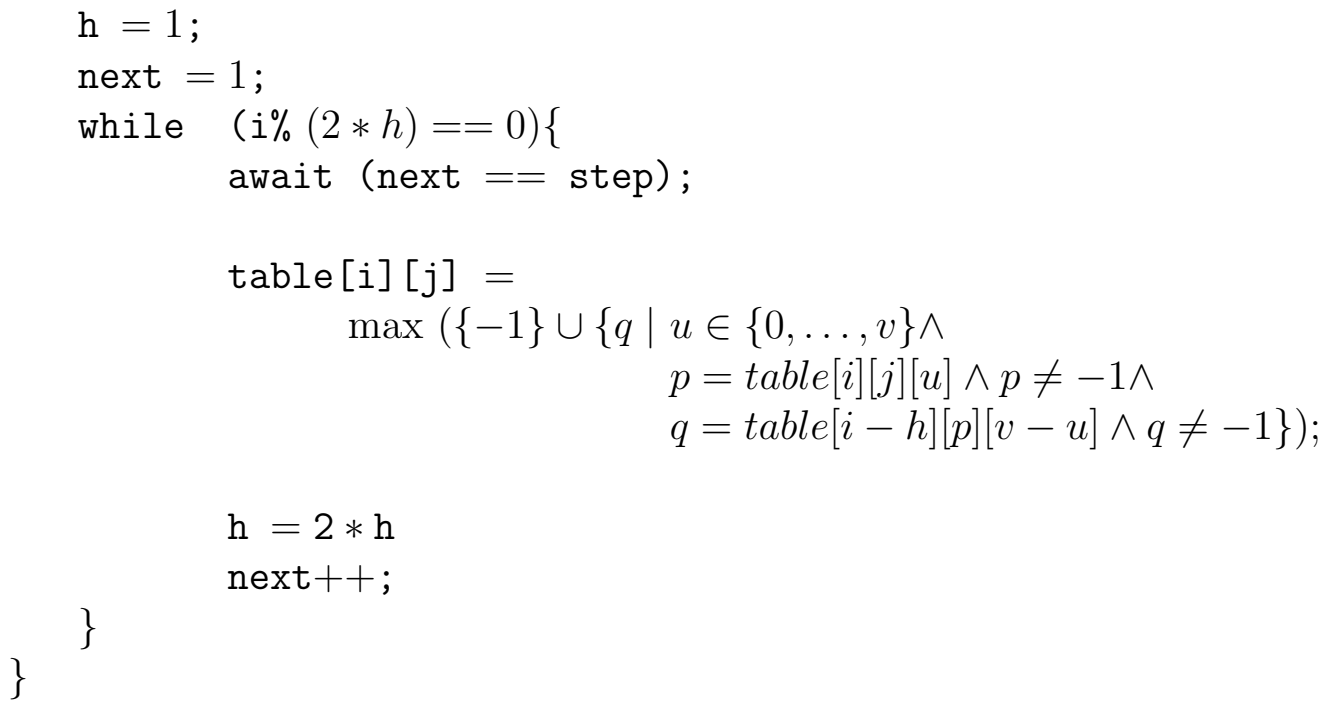

For the purpose of giving a correctness proof, we now formulate and prove the invariant, which was outlined intuitively before presenting the algorithm.

Lemma 1 The following loop invariant holds for the values of step encountered in the algorithm:

for $i \in\left\{c \cdot 2^{\text {step }}\left|c \in \mathbb{N} \backslash\{0\} \wedge c \cdot 2^{\text {step }} \leq\right| s \mid\right\}$, and $j, v \in\{0, \ldots,|t|\}$, we have that table $[i][j][v]$ equals

$$
\max \left(\{-1\} \cup\left\{r \mid 0 \leq r \leq j \wedge \operatorname{count}\left(s_{i-2^{s t e p}+1} \cdots s_{i}, t_{r+1} \cdots t_{j}\right)=v\right\}\right) .
$$

Proof First, we prove that the invariant holds after the initialization. As step $=0$, we consider all $i$ 's in $\{1, \ldots,|s|\}$. In the following, assume that $i$ and $j$ are fixed. Notice that $s_{i-2^{s t e p}+1} \cdots s_{i}$ is simply $s_{i}$. For each $v$, we determine the maximal $r$ such that $r \leq j$ and $\operatorname{count}\left(s_{i}, t_{r+1} \cdots t_{j}\right)=v$.

Assume that $v=0$. As count $\left(s_{i}, \varepsilon\right)=0$, we can choose $r=j$ ( $\varepsilon$ denotes the empty string).

Assume that $v=1$. In order for $\operatorname{count}\left(s_{i}, t_{r+1} \cdots t_{j}\right)$ to equal one, $s_{i}$ must appear in $t_{1} \cdots t_{j}$. If it does not, then the maximum is -1 and table $[i][j][1]$ is also assigned -1 . Now, assume that $s_{j}$ is present in $t_{1} \cdots t_{j}$. Let $t_{u}$ be the right-most occurrence of $s_{i}$ in $t_{1} \cdots t_{j}$. Then $\operatorname{count}\left(s_{i}, t_{u} \cdots t_{j}\right)=1$ and $u$ is the maximal integer for which this is the case. So, we can choose $r=u-1$. 
Assume that $v>1$. As $s_{i}$ has length one, $\operatorname{count}\left(s_{i}, t_{r+1} \cdots t_{j}\right)$ can be at most one no matter what the value of $r$ is. So, in this case, table $[i][j][u]$ should be assigned -1 .

Now, we turn to the induction step. Let $s t e p^{\prime}=$ step +1 . Assume that the maximum is -1 , i.e., no $r$ exists such that

$$
\operatorname{count}\left(s_{i-2^{\text {step }}+1} \cdots s_{i}, t_{r+1} \cdots t_{j}\right)=v \text {. }
$$

This means that even $\operatorname{count}\left(s_{i-2^{\text {step }}+1} \cdots s_{i}, t_{1} \cdots t_{j}\right)<v$. Now, assume to the contrary that a $u$ exists such that $p \neq-1$ and $q \neq-1$. By the invariant, this means that $\operatorname{count}\left(s_{i-2^{\text {step }} p_{1}} \cdots s_{i}, t_{p+1} \cdots t_{j}\right)=u$ and that $\operatorname{count}\left(s_{i-2^{s t e p^{\prime}}+1} \cdots s_{i-2^{\text {step }}}, t_{q+1} \cdots t_{p}\right)=v-u$. But the two substrings of $s$ are disjoint as are the two substrings of $t$, so by definition of count, we must have that $\operatorname{count}\left(s_{i-2^{s t e p^{\prime}}+1} \cdots s_{i}, t_{q+1} \cdots t_{j}\right)=v$, which is a contradiction.

Now, assume that the maximum is $r \geq 0$. By dividing a maximal common subsequence of $s_{i-2^{s t e p^{\prime}}+1} \cdots s_{i}$ and $t_{r+1} \cdots t_{j}$ up into the part which is in $s_{i-2^{s t e p^{\prime}}+1} \cdots s_{i-2^{\text {step }}}$ and the part in $s_{i-2^{s t e p^{\prime}+1}} \cdots s_{i}$ it is clear that a $u$ and an $l$ must exist such that

$$
\operatorname{count}\left(s_{i-2^{s t e p}+1} \cdots s_{i}, t_{l+1} \cdots t_{j}\right)=u
$$

and

$$
\operatorname{count}\left(s_{i-2^{s t e p^{\prime}}+1} \cdots s_{i-2^{s t e p}}, t_{r+1} \cdots t_{l}\right)=v-u
$$

By the invariant, $p$ (in the algorithm) is the maximal integer such that $\operatorname{count}\left(s_{i-2^{\text {step }}+1} \cdots s_{i}, t_{p+1} \cdots t_{j}\right)=u$, so obviously $l \leq p$. But then

$$
\operatorname{count}\left(s_{i-2^{\text {step }}+1} \cdots s_{i-2^{\text {step }}}, t_{r+1} \cdots t_{p}\right)
$$

is at least $v-u$. By the invariant, $q$ (in the algorithm) is now the maximal integer such that count $\left(s_{i-2^{s t e p^{\prime}}+1} \cdots s_{i-2^{\text {step }}}, t_{q+1} \cdots t_{p}\right)=v-u$. So, given that we insist on matching exactly $u$ elements from $s_{i-2^{\text {step }}+1} \cdots s_{i}$ with elements from $t, q$ must also be the maximal integer such that $\operatorname{count}\left(s_{i-2^{s t e p}{ }^{\prime}+1} \cdots s_{i}\right.$, $\left.t_{q+1} \cdots t_{j}\right)=v$. Because in order to use a larger $q$, we would have to use a larger $p$, and that would make 


$$
\operatorname{count}\left(s_{i-2^{\text {step }}+1} \cdots s_{i}, t_{p+1} \cdots t_{j}\right)
$$

strictly smaller than $u$, as $p$ was chosen to be maximal.

As all $u$ 's are considered in the algorithm, and as $s_{i-2^{s t e p}+1} \cdots s_{i}$ must match $u$ elements from $t$ for some $u(0 \leq u \leq v)$, we are bound to find the right $u$ and, thus, the correct maximum value.

Corollary 2 Algorithm Fast Counting correctly computes count(s,t) when the two strings $s$ and $t$ are used in the algorithm and the final result is computed from the table by

$$
\operatorname{count}(s, t)=\max _{0 \leq v \leq m}\{v \mid \text { table }[k][m][v] \neq-1\} .
$$

Proof The last processors terminate when step $=\log k$, and at that point,

$$
\text { table }[k][m][v]=\max \left(\{-1\} \cup\left\{r \mid 0 \leq r \leq m \wedge \operatorname{count}\left(s, t_{r+1} \cdots t_{m}\right)=v\right\}\right) .
$$

Clearly, table $[k][m][v]=-1$ if and only if $\operatorname{count}(s, t)<v$.

Finally, we consider the complexity issues. Recall that in the CREW PRAM model, there are a polynomial number of processors and a shared memory. Any number of processors can read from the same memory cell at the same time, but at most one processor can be writing to any memory cell at a given time. The COMMON model is a CRCW PRAM model. The CRCW PRAM is like the CREW PRAM except that concurrent writes are allowed. In the COMMON model, if more than one processor writes to the same memory cell at the same time, then they must all write the same value. The COMMON model has also been called a WRAM in the literature.

The algorithm has been designed such that maxima computations and the remaining computation can be analyzed separately. In the following, we state the known maxima results of interest here.

Lemma 3 The problem of calculating the maximum of $n$ elements is in NC, and the maximum can be computed in time

- $O(\log n)$ in the CREW model.

- $\Theta(1)$ in the COMMON CRCW model. 
Proof In the CREW model, use a binary tree of height $\lceil\log n\rceil$.

In the COMMON model, $\left(\begin{array}{l}n \\ 2\end{array}\right)$ processors are used to perform every possible comparison in one step. The details can be found in [3].

Only comparisons, assignments, additions, and subtractions are used in the algorithms, so the problem is clearly in NC.

Theorem 4 The complexity of calculating the length of the maximal common subsequences is

- $O(\log |t| \cdot \log |s|)$ in the CREW model.

- $\Theta(\min (\log |t|, \log |s|))$ in the COMMON model.

Proof Rename, if necessary, so that $|s| \leq|t|$. The algorithm runs in $\log |s|+1$ steps and, except for the calculation of maxima, only a constant amount of work is done by each processor in each step. Notice that the sets for which we find the maxima have size at most $|t|+2$. Furthermore, the predicates used in their definition can be computed in constant time. The result now follows from lemma 3 .

Corollary 5 The problem of computing the length of the maximal common subsequences is in $\mathrm{NC}$.

Proof All computations, except the maxima computations, are independent of the input length. As stated in lemma 3, computing the maximum is in $\mathrm{NC}$, so our problem is as well.

Notice that since computing the maximum is in $\mathrm{NC}^{1}$, the problem of computing the length of the maximal common subsequences is in $\mathrm{NC}^{2}$. Using unbounded fan-in, the maximum can be computed in constant time, so the problem of computing the length of the maximal common subsequences is also in $\mathrm{AC}^{1}$.

Acknowledgement The author would like to thank Joan Boyar for valuable discussions on the models of parallel computation and for helpful comments on an earlier draft of the paper. 


\section{References}

[1] Alfred V. Aho. Algorithms for finding patterns in strings. In J. van Leeuwen, editor, Handbook of Theoretical Computer Science, pages 255300. Elsevier Science Publishers, 1990.

[2] D. S. Hirschberg. A linear space algorithm for computing maximal common subsequences. Comm. ACM, 18(6):341-343, 1975.

[3] Yossi Shiloach and Uzi Vishkin. Finding the maximum, merging, and sorting in a parallel computation model. J. Algorithms, 2:88-102, 1981.

[4] Graham A. Stephen. String search. Technical Report TR-92-gas-01, School of Electronic Engineering Science, University College of North Wales, 1992. 